\title{
Can Antidepressants Act as Potential Pro-neoplastic Agents in Patients with Neurotrophic Factor-Related Cancers?
}

To the Editor:

April 28, 2008

The prevalence of major depressive disorder is estimated to be $40 \%$ in patients with cancer, and this is a 2- to 4-times higher frequency than that in the general population. ' Long-term, adequate antidepressant treatment is thought to be the most effective way to treat cancer-related depression, and the response rate has been reported as high as $80 \% .{ }^{2}$ Although antidepressants have long been accepted as efficacious and safe treatments, some potential adverse effects may be overlooked and we would like to remind clinicians to remain vigilant for potential adverse effects when prescribing antidepressants for cancer patients.

The therapeutic mechanism of antidepressants centers on the restoration of deficits in depression-associated neurogenesis through an increase in growth factors, and this is considered to hold for most of the contemporary antidepressive agents..$^{3,4}$ Out of all of the recognized growth factors, brain-derived neurotrophic factor (BDNF) and its receptor, the tropomyosin receptor kinase $B$ (TrkB), may play a central role in the pathogenèsis of major depressive disorder and the therapeutic mechanism of antidepressants.

In rodent models, repeated administration of an antidepressant resulted in elevated levels of BDNF mRNA and associated proteins in several brain regions, namely the hippocampus and prefrontal cortex. ${ }^{5,6}$ Chronic use of antidepressants also elicited the expression and activation of TrkB in the same areas. ${ }^{4,7}$ In humans, it has been determined that BDNF expression was increased in the hippocampus of subjects treated with antidepressant medication at the time of death, compared with untreated controls. ${ }^{8}$ It is believed that most antidepressants, despite their primary therapeutic class of action, share an ability to augment BDNF production and up-regulate TrkB signaling.
Given that the BDNF-TrkB pathway acts as a potent neurotrophic signaling system through neuronal maturation, this signaling cascade has been suspected and subsequently confirmed in the tumorinogenesis of various cancers of neuroectodermal origin (eg, neuroblastoma, glioneuronma, astrocytoma, ependymoma, ganglioneuroma, paraganglioma, phaeochromocytoma, and melanoma).., 10

BDNF and TrkB also contribute to survival cues and rescue from chemotherapeutic agent-induced cell death in neuroblastoma cells in vitro through activation of the phosphatidylinositol-3 kinase protein kinase $B$ signaling cascades. For example, the addition of BDNF protects human neuroblastoma cell lines from apoptosis induced by cisplatin, doxorubicin, etoposide, and vinblastine. ${ }^{11}$

Given that antidepressants are responsible for the enhancement of BDNF-TrkB signaling and this pathway has been implicated in several types of malignancies, we hereby hypothesize that antidepressants may act as potential pro-neoplastic agents and therefore actually worsen prognosis in patients with neurotrophic factor-related cancers. In addition, although this hypothesis has been formulated on the basis of the BDNF-TrkB cascade, recent work has also suggested that vascular endothelial growth factor may also contribute an antidepressant effect. ${ }^{12}$ Vascular endothelial growth factor is notoriously involved in angiogenesis and tumorigenesis, ${ }^{13}$ it may also be implicated in an antidepressant-induced cancer risk and this should also be considered. Supporting evidence for this hypothesis may come from Brandes and colleagues, ${ }^{14}$ who reported that antidepressants, fluoxetine and amitriptyline, can promote growth of melanoma in rodents at clinically relevant doses.

Given that antidepressants are so commonly used in cancer patients, it is of significant public health importance that their effect on tumor recurrence rates, tumor size and degree of 


\section{Communique}

metastasis is fully explored in patients with neurotrophic factor-related cancers.

Sincerely,

Yung-Tian A. Gau, MD

Chen-Jee Hong, MD

Shih-Jen Tsai, MD

\section{REFERENCES}

\section{Massie MJ. Prevalence}

Monogr. 2004:32:57-71

2. Chaturvedi S Maguire P Hopwood P. Antidepressant medications in cancer patients. Psycho-Oncology. 1994;3:57-60.

3. Dias BG, Banerjee SB, Duman RS, Vaidya VA. Differential regulation of brain derived neurotrophic factor transcripts by antidepressant treatments in the adult rat brain. Neuropharmacology. 2003:5:553-563.

4. Nibuya M, Morinobu S, Duman RS. Regulation of BDNF and trkB mRNA in rat brain by chronic electroconvulsive seizure and antidepressant drug treatments. J Neurosci. 1995:15:7539-7547

5. Ivy AS, Rodriquez FG, Garcia C, Chen MJ, Russo-Neustadt AA. Noradrenergic and serotonergic blockade inhibits BDNF mRNA activation following exercise and antidepressant. Pharmacol Biochem Behav. 2003;75:81-88.

6. Coppell AL, Pei Q, Zetterstrom TSC. Bi-phasic change in BDNF gene expression follow ing antidepressant drug treatment. Neuropharmacology. 2003:44:903-910.

7 Saarelainen T, Hendolin P. Lucas G, et al. Activation of the TrkB neurotrophin receptor is induced by antidepressant drugs and is required for antidepressant induced behavioral effects. J Neurosci. 2003:23:349-357.

8. Chen B, Dowlatshahi D. MacQueen GM, Wang JF, Young LT. Increased hippocampal BDNF immunoreactivity in subjects treated with antidepressant medication. Biol Psychiatry. 2001:50:260-265

9. Sartor L, Negro A, Barletta E, Mugnai G, Garbisa S. Modulation of proteolytic potential and differentiation by CNTF and BDNF in two mouse neuroblastoma clones: relation to invasion. Clin Exp Metastasis. 2002;19:709-716.

10. Middlemas DS, Kihl BK, Moody NM. Brain derived neurotrophic factor protects human neuroblastoma cells from DNA damaging agents. J Neurooncol. 1999:45:27-36.
11. Jaboin J Kim CJ, Kaplan DR, Thiele CJ. Brain-derived neurotrophic factor activation of TrkB protects neuroblastoma cells from chemotherapy-induced apoptosis via phosphatidylinositol 3'-kinase pathway. Cancer Res. 2002;62:6756-6763.

12. Warner-Schmidt JL, Duman RS. VEGF is an essential mediator of the neurogenic and behavioral actions of antidepressants. Proc Natl Acad Sci U S A. 2007:104:4647-4652.

13. McMahon G. VEGF receptor signaling in tumor angiogenesis. Oncologist. 2000:5(suppl 1):3-10

14. Brandes LJ, Arron RJ, Bogdanovic RP, et al. Stimulation of malignant growth in rodents by antidepressant drugs at clinically relevant doses. Cancer Res. 1992;52:3796-3800.

Dr. Gau is master student at the School of Medicine at National Yang-Ming University in Taipei, Taiwan. Dr. Hong is associate professor at the School of Medicine at National Yang-Ming University, is attending psychiatrist in the Department of Psychiatry at Taipei Veterans General Hospital, and associate professor at the Institute of Brain Science at National Yang-Ming University. Dr. Tsai is associate professor at the School of Medicine at National Yang-Ming University and attending psychiatrist in the Department of Psychiatry at Taipei Veterans General Hospital.

Disclosures: The authors do not have an affiliation with or financial interest in any organization that might pose a conflict of interest.

Please send letters to the editor to: CNS Spectrums, c/o Eric Hollander, MD, 333 Hudson St., 7th Floor, New York, NY 10013; E-mail: vi@mblcommunications.com.

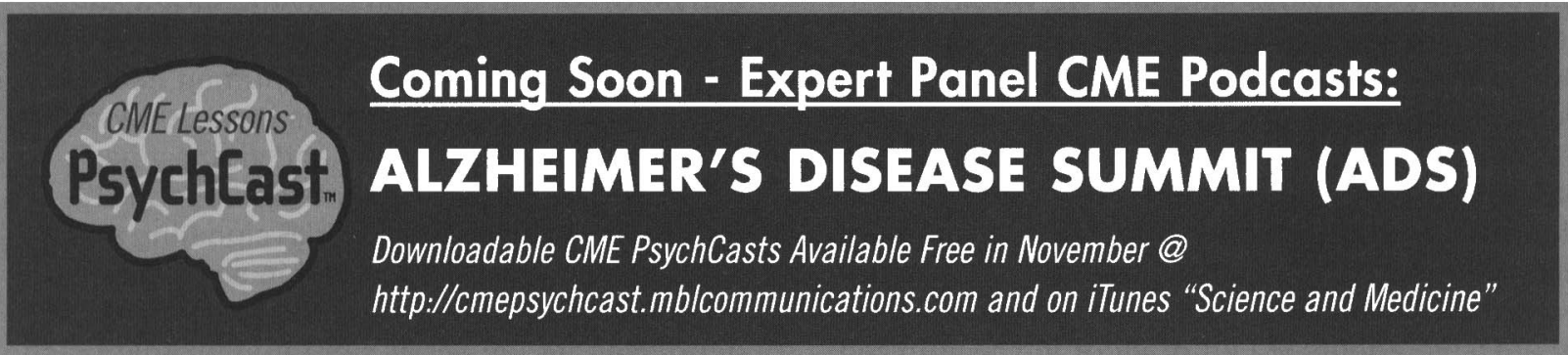

Jointly sponsored by The Mount Sinai School of Medicine and MBL Communications, Inc.

\section{"Advances in Clinical Assessment"}

Ziad Nasreddine, MD, Kathleen A. Welsh-Bohmer, PhD, and Ellen Woo, PhD

\section{"Advances in Neuroimaging and Biomarkers"}

Liana G. Apostolova, MD, Mark A. Mintun, MD, and Elaine R. Peskind, MD

\section{"Current Alzheimer's Disease Therapy: Best Practices"}

Jeffrey L. Cummings, MD, Gary W. Small, MD, and Rachelle S. Doody, MD, PhD

\section{"The Future of Alzheimer's Disease Therapeutics"}

Paul S. Aisen, MD, Norman R. Relkin, MD, PhD, and Mary Sano, PhD

Funding for these podcasts has been provided by educational grants from

Forest Pharmaceuticals, Inc., Eisai Inc., Medivation, Inc., and Elan Pharmaceuticals, Inc.

commumiations PRIMARY PSYCHIATRY CNS SPECTRUMS PsychiatryWeekly.

$\mathrm{ADS}$ 


\section{On-demand CME is just a few clicks away.}

\section{Members have access to over 100 hours of no-cost credit online.}

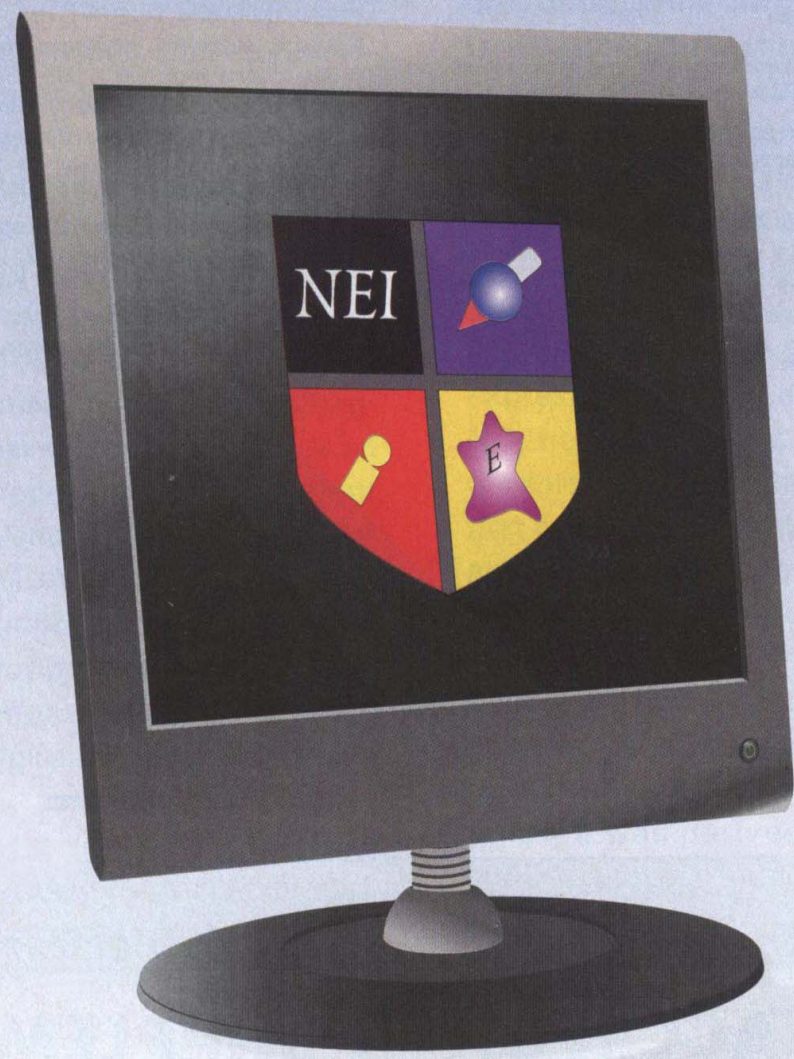

For convenient access to the latest peer-reviewed learning opportunities and AMA PRA Category $1 \mathrm{Credit}^{T M}$

ADD/ADHD

Alcohol/Substance

Abuse

Anxiety

Bipolar Disorder

CATIE
Children/Adolescents

Dementia

Depression

Fatigue

Metabolic

Mood
Pain

PTSD

Psychosis

Schizophrenia

Sleep

Stress

\section{Visit us today at www.neiglobal.com}

